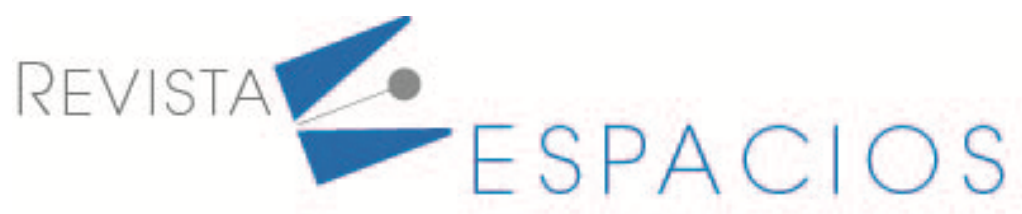

\title{
Influencia del ambiente áulico en la motivacion de los estudiantes para el aprendizaje de un curso de analisis de alimentos
}

\section{Influence of the classroom environments on the Motivation of Students to Learn the Food Analysis Course}

\author{
TARÓN DUNOYER, Arnulfo ${ }^{1}$ \\ DIAZ CABALLERO, Antonio ${ }^{2}$ \\ ALVIZ AMADOR, Antistio ${ }^{3}$
}

\begin{abstract}
Resumen
En esta investigación se estudia la influencia del ambiente áulico, en la motivación y el rendimiento académico. Se realiza un estudio descriptivo-cuantitativo, de dos ambientes áulicos (motivado y no motivado), utilizando un instrumento de evaluación tipo Liker de 15 dimensiones, de escala unipolar de criterios numéricos, de (1) a (5). Los estudiantes motivados, obtuvieron mayor rendimiento académico promedio $(3,98)$, que los no motivado $(3,37)$, encontrando diferencias estadísticamente significativas a $(p<0,001$. Un ambiente áulico motivante, facilita obtener alto rendimiento académico.

Palabras clave: ambiente áulico, aprendizaje, motivación, rendimiento académico

Abstract

In this research, the influence of the classroom environment on motivation and academic performance is studied. A descriptive-quantitative study of two classroom environments (motivated and unmotivated) was carried out, using a 15-dimensional Liker-type assessment instrument, with a unipolar scale of numerical criteria, from (1) to (5). Motivated students obtained higher average academic performance (3.98) than non-motivated students (3.37), finding statistically significant differences at ( $p$ $<0.001$. A motivating classroom environment facilitates obtaining high academic performance.

Keywords: academic performance, classroom environment, learning, motivation
\end{abstract}

\section{Introducción}

Uno de los aspectos fundamentales en el proceso enseñanza aprendizaje, es la motivación. Gran parte de los fracasos en este proceso, tiene su origen en un posicionamiento inicial afectivo, totalmente destructivo de sus propias potencialidades, que es provocado, en muchos casos, por la inadecuada introducción por parte del docente del curso respectivo (Lewthwaite and Wulf, 2017). La motivación de los alumnos es muy relevante y es

\footnotetext{
${ }^{1}$ Docente Titular. Facultad de Ingeniería. Universidad de Cartagena. atarond@unicartagena.edu.co

${ }^{2}$ Docente Titular. Facultad de odontología. Uiversidd de Cartagen. adiazc1@unicartagena.edu.co

${ }^{3}$ Docente Asistente. Facultad de Farmacia. Universidad de Cartagena. aalviza@unicartagena.edu.co
} 
aceptada, como un factor determinante, que tiene mucha influencia en el éxito y la rapidez del aprendizaje. De hecho, la motivación es un importante componente para que los estudiantes alcancen el éxito en cualquier ámbito de aprendizaje (Sukor et al., 1027; Bukhari et al., 1014). Existe una estrecha relación entre el deficiente rendimiento académico por parte de los estudiantes y algunos factores como la falta de motivación, la carencia de estrategias de estudios adecuadas y su necesidad de tener una guía por parte de sus docentes y por lo tanto, limitarse a sus propias capacidades y motivaciones (Hernández et al., 2017; Wouters et al., 2016; Zürcher et al., 2010).

Algunos estudios indican que, los enfoques de aprendizaje de los estudiantes pueden cambiar y ser afectados por su atributos personales individuales y contextos de aprendizaje (Hall et al., 2004), de ahí que, los estudiantes adaptan su aprendizaje de acuerdo con sus percepciones del ambiente áulico (Poondej \& Lerdpornkulrat, 2016).

En algunas investigaciones se ha intentado identificar las variables y los entornos de aprendizaje que tienden a compensar la falta de motivación y atención en el aprendizaje presentadas por algunos estudiantes. Siguiendo ajuste de escenario-ambiente, según la teoría, una contribución importante a estos descensos en la motivación del estudiante y el autoconcepto en la adolescencia se atribuye a una falta de adecuación del desarrollo del individuo a sus necesidades y al entorno educativo proporcionado (Kulakow, 2020; Ecles \& Midgley, 1989). Por lo tanto, para apoyar eficazmente a los estudiantes en su educación, es imprescindible examinar los entornos de aprendizaje y las características que respaldan el desarrollo motivacional positivo (Lazarides \& Reulfelder, 2017; Bakadorova \& Raufelder, 2016).

Los estudiantes universitarios en muchas ocasiones por falta de motivación y atención, tienen que lidiar con una disminución de su rendimiento académico, durante el desarrollo de su carrera académica. Muy pocos, son los que saben aprovechar las oportunidades de aprendizaje a partir de estas situaciones. Una de las maneras en las que los estudiantes pueden mejorar su aprendizaje después de experimentar deficiencias en su rendimiento, es adaptando su comportamiento de aprendizaje, reflexionando sobre los conceptos erróneos subyacentes. Además de estas actividades (meta) cognitivas y acciones de aprendizaje, los estudiantes tienen que lidiar con elementos motivacionales y afectivos negativos (Reindl et al., 2020).

Tres aspectos de la motivación que pueden versos afectados frecuentemente en los estudiantes son la autoeficacia, la competencia percibida y el interés del tema. Se ha detectado que la autoeficacia, puede tener un efecto positivo sobre factores como: la motivación académica, el comportamiento de estudio y en los resultados de aprendizaje (Harsel et al., 2019; Orsini et al., 2015).

El objetivo de la presente investigación fue analizar la influencia del ambiente áulico de aprendizaje, en la motivación y en el rendimiento académico de estudiantes del curso de análisis de alimentos de un programa de Ingeniera de Alimentos, de una universidad pública de Colombia

\section{Metodología}

\subsection{Sujetos}

Estudiantes del programa de Ingeniería de alimentos de la Facultad de Ingeniería de una universidad pública en la ciudad de Cartagena. Para la realización de este estudio, a cada uno de los estudiantes partícipes se les informó con detalles a través de un formato de consentimiento informado, del tipo de investigación a realizar, sus objetivos y posibles aplicaciones.

\subsection{Población de estudio}

Esta investigación es de tipo descriptivo, con enfoque cuantitativo, donde se utilizó como objeto de estudio dos grupos heterogéneos (hombres y mujeres) de 40 estudiantes del curso de análisis de los alimentos, de un 
programa de ingeniería de alimentos de una universidad estatal en Cartagena, a los cuales se le evaluó el rendimiento académico, con base en el aprendizaje de una temática inmersa en la asignatura de análisis de alimentos, en dos ambientes áulicos diferentes,

Las edades de los estudiantes estaban entre los 19 y 21 años. No debían tener antecedentes académicos negativos en sus registros académicos.

En el cuadro 1 se describen las características demográficas de los grupos objeto de estudio.

Cuadro 1

Características demográficas

de la población estudiantil

\begin{tabular}{|c|c|c|c|c|c|}
\hline \multicolumn{6}{|c|}{ Características y condiciones de la población estudiantil } \\
\hline Grupo estudiantil & $\begin{array}{l}\text { Ambiente } \\
\text { aprendizaje }\end{array}$ & de & Estudiantes hombre & Estudiantes mujeres & $\begin{array}{l}\text { Edad } \\
\text { promedio(años) }\end{array}$ \\
\hline Grupo 1 & No motivados & & 12 & 8 & 20 \\
\hline Grupo 2 & Motivados & & 11 & 9 & 20 \\
\hline
\end{tabular}

Fuente: los autores

\subsection{Ambiente áulico de aprendizaje}

Se utilizaron dos ambientes áulicos diferentes para el desarrollo del proceso de enseñanza-aprendizaje. Un grupo de estudiantes recibió las clases de la asignatura de análisis de alimentos, en un aula con nivel adecuado de: ruido, temperatura, iluminación, sillas cómodas, además de un espacio amplio y con las herramientas pedagógicas necesarias para facilitar la conexión teórico-práctica. En este ambiente el docente explica con detalles la metodología, objetivos y aplicación práctica de los saberes a impartir. Los estudiantes de este ambiente tenían dinámica y posibilidad de interacción grupal. El otro ambiente fue de tipo tradicional, no motivado, además de no haber interacción dinámica e interacción grupal, con espacio físico reducido, poca ventilación, con nivel de ruido no adecuado.

\subsection{Efecto del ambiente áulico en la motivación para el aprendizaje}

Se utilizó como instrumento de medición del efecto del ambiente áulico en la motivación para el aprendizaje (IAAMA), un cuestionario de quince (15) interrogantes (dimensiones), tipo Likert de escala unipolar, el cual fue aplicado a la población estudiantil objeto de estudio. Se utilizó el estadígrafo T-Student para obtener datos comparativos estableciendo diferencias entre grupos aceptando diferencias significativas a un valor de $p<0.001$. En el instrumento que aparece como anexo 1 en la sección de anexos de este manuscrito, se puede apreciar las preguntas (dimensiones) realizadas en el instrumento de evaluación. Para analizar el efecto del ambiente áulico en la motivación para el aprendizaje. Se utilizó como variable de respuesta el rendimiento académico, el cual se sustenta en la calificación, con base en una escala de valores de cinco (5) criterios, de 1 a 5 puntos, siendo cinco (5) la máxima y uno (1) la mínima. Los resultados del instrumento de medición IAAMA, se expresan en términos de porcentajes de acorde a los criterios de valores encontrados para cada dimensión, evaluando la desviación estándar, el efecto techo y el efecto suelo, en el grupo de estudiantes motivados (MG) y no motivados(NMG).

\section{Resultados}

En la figura 1 se puede apreciar el rendimiento académico obtenido por los grupos estudiantiles objeto de estudio en esta investigación, resaltando que, el grupo de los estudiantes motivados (AAM) presentó un rendimiento académico superior al grupo de los estudiantes no motivados (AANM), para los cuales se 
encontraron, diferencias estadísticamente significativas a $p<0,001$, entre sus valores promedios. El valor medio de las calificaciones para el grupo de estudiantes motivados fue de 3,98 puntos y para los estudiantes no motivados fue 3,37 puntos. El valor máximo y mínimo del rendimiento académico, de los estudiantes del ambiente áulico motivado (AAM) fue de 4,2 y 3,7 puntos respectivamente, mientras que para los del ambiente áulico no motivado(AANM) fueron de 4,0 y 3,0 puntos respectivamente. De acuerdo a estos resultados, es importante resalta la relevancia que tiene, desarrollar e impartir los contenidos académicos en ambientes áulicos adecuados, que permitan motivar a los estudiantes y así de esta forma el proceso enseñanza-aprendizaje se desarrolle de manera óptima, y poder obtener rendimientos superiores.

Figura 1

Rendimiento académico de los estudiantes en ambientes áulicos motivados y no motivados

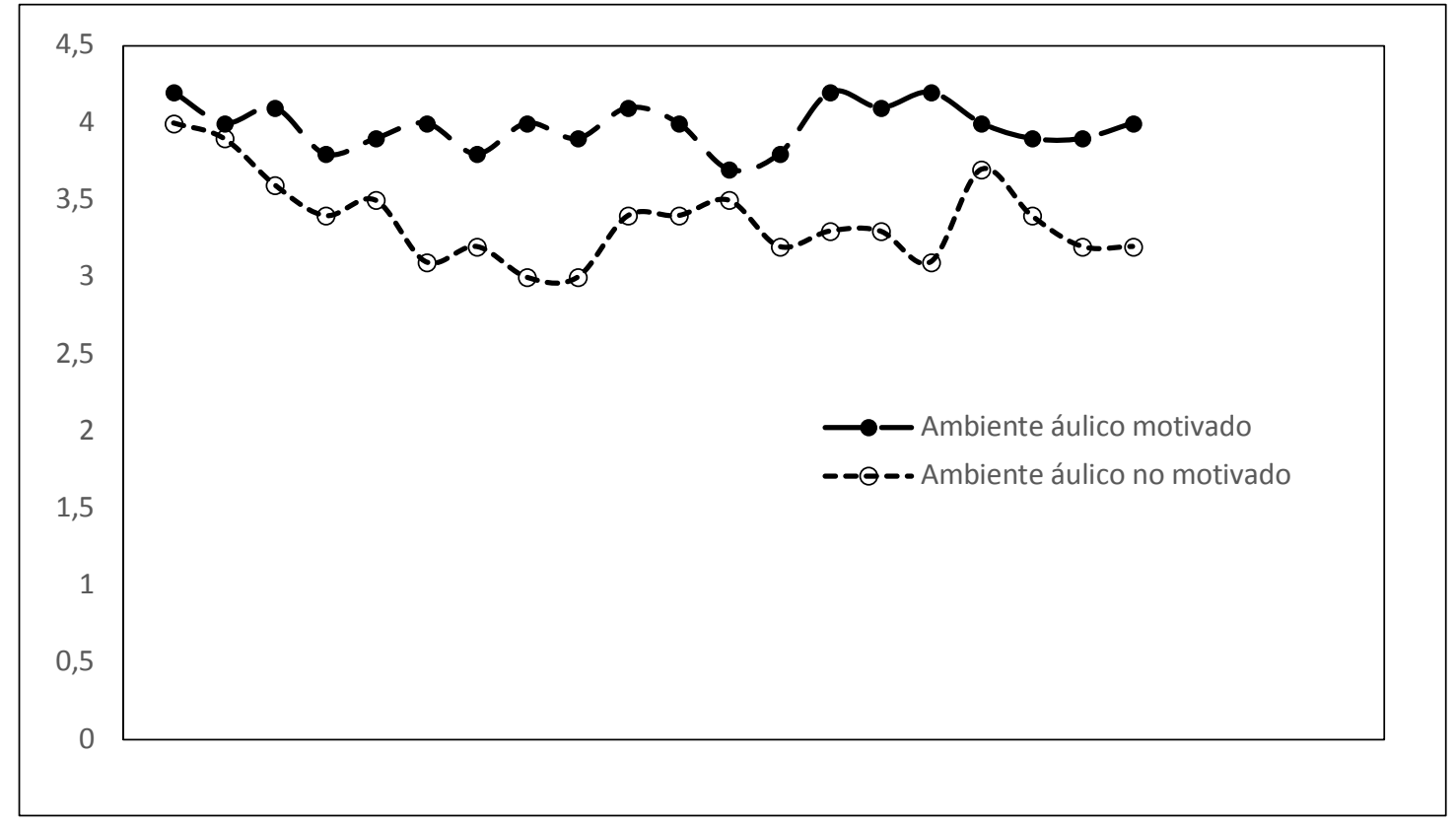

Para lograr que el ambiente áulico tuviera un efecto positivo y motivacional en los estudiantes fue indispensable cumplir con algunas características que garantizaron una buena actitud para el proceso enseñanza-aprendizaje, entre las que se destacan: aulas ordenadas, aseadas, cómodas, bajo nivel de ruido, interacción grupal de tal forma que el alumno pudiera experimentar sensaciones de bienestar físico, la cual permite la aceptación de muchos estímulos.

Este ambiente motivado, influye positivamente en la confianza de los estudiantes, les permitió estar a gusto emocional y físicamente en el lugar donde se llevó a cabo el aprendizaje (Duarte, 2003).

\subsection{Ambiente Áulico Motivado (AAM)}

En la figura 2, se muestran las calificaciones obtenidas (\%) para cada una de las dimensiones estudiadas en este trabajo, acorde a estos resultados, el $95 \%$ de los estudiantes considera que el ambiente áulico fue motivacional (Qu3: ¿Considera que el ambiente áulico es motivacional para el aprendizaje de la asignatura?), para el desarrollo del proceso enseñanza -aprendizaje.

Hay que resaltar que el 85\% de los estudiantes otorga la máxima calificación (5) a la dimensión Qu5 (¿sintió ganas de estar en el aula recibiendo los contenidos de la asignatura?), esta misma calificación se obtiene para Qu10 (¿considera que el espacio del aula es amplio y adecuado?) y Qu14 (¿considera que la interacción grupal, lo motiva para el aprendizaje?) en las que se obtiene un 90 y $85 \%$ respectivamente. Para la dimensión Qu6 (¿le gustó las herramientas y ayudas didácticas utilizadas por el docente?), el 60\% de los estudiantes le da la máxima 
calificación y un 35\% la da la calificación de 4 en la escala, es decir el 95\% de los estudiantes estuvo identificado a plenitud con las herramientas y ayudas utilizadas por el docente. Con respecto a la dimensión Qu8 (¿la clase fue interesante y le dieron ganas de participar durante el desarrollo de esta?) y Qu11 (¿la temperatura dentro del aula fue agradable?) presentaron resultados superiores al 70\%, dándole una calificación de 5 puntos en la escala de valores, (Qu8:80\%) y (Qu11:70\%).

Teniendo en cuenta que la dimensión Qu15(¿considera que el ambiente áulico es el a adecuado?), es una de las de mayor relevancia, se resalta que el $60 \%$ de estudiantes, la califican con el valor máximo en la escala y un 30 $\%$ con 4 . Solo un $10 \%$ de los estudiantes considera que el ambiente áulico fue medianamente adecuado. El $95 \%$ de los estudiantes manifiesta que el nivel de ruido (Qu7: ¿el nivel de ruido dentro del aula causó alguna distracción?) en el aula fue adecuado, de tal forma que no fue un elemento distractor durante el desarrollo de la asignatura. Así mismo, los resultados referidos a la dimensión Qu5 (¿sintió sudoración durante el desarrollo de la clase?) presentó resultados positivos, indicando que la temperatura dentro del aula fue agradable, para Qu5, el $50 \%$ de los estudiantes, califican esta dimensión con un valor de 4 y 5 puntos en la escala.

Todos estos resultados encontrados para el grupo poblacional de estudiantes motivados, se corresponden con un indicador de rendimiento académico superior, con respecto al grupo de estudiantes en un ambiente áulico no motivado. El rendimiento académico del grupo motivado, presento diferencias significativas $(p<0,001)$, con respecto al grupo de estudiantes no motivados.

Figura 2

Resultado del cuestionario dimensional

para el ambiente áulico motivado (AAM).

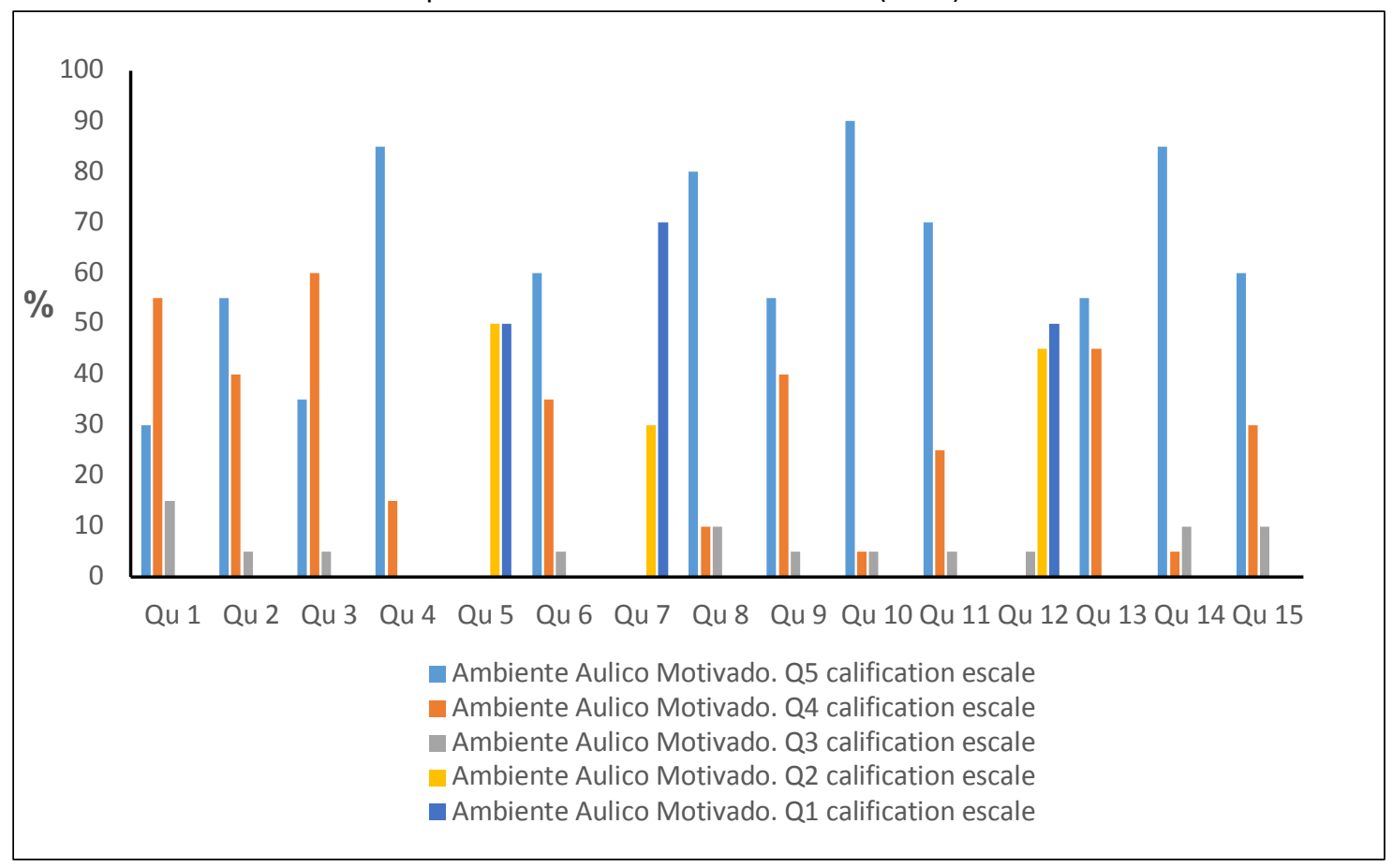

\subsection{Ambiente Aúlico no motivado (AANM)}

En la figura 3, se muestran los resultados (\%) del grupo poblacional no motivado, se observa que el 55\% de los estudiantes considera que la clase le agradó medianamente (Qu1) con una calificación de 3 puntos en la escala y $30 \%$ le da una puntuación del 4, ninguno de los estudiantes le dio una calificación de 5 a esta dimensión, estos 
resultados están en contraposición con respecto a los encontrados en el grupo poblacional de estudiantes motivados

El 90\% de los estudiantes manifiesta no haberse sentido cómodo en el desarrollo de la clase (Qu2). Para el 80\% de los estudiantes la temperatura dentro del aula no fue la adecuada (Qu5), y manifestaron sentir un alto grado de sudoración, para lo cual le asignan una calificación de 4 puntos (60\%) y 5 puntos (20\%) respectivamente.

En la dimensión Qu12, el 60 \% de los estudiantes manifiesta la existencia de un nivel de ruido alto dentro del aula, lo que incide entre tantos factores a una disminución del rendimiento académico. Para la dimensión Qu3, los estudiantes consideran que el ambiente, fue medianamente motivacional (60\%), solamente el $30 \%$ de este grupo le da una calificación de 5 puntos. Con relación a la dimensión Qu13 (¿existió interacción grupal con sus compañeros de aula?, el 65\% manifiesta que no existió interacción grupal. Para Qu9 (¿le gustó la dinámica de la clase?), se puede decir que el $70 \%$ de los alumnos no se encuentran a gusto con la dinámica desarrollada para la asignatura. En la dimensión Qu15 (¿considera que el ambiente áulico es el adecuado?) los estudiantes consideran que el ambiente áulico no es el adecuado para motivar al aprendizaje (65\%). Los resultados permiten inferir que este grupo no estaba motivado para el aprendizaje, y esto puede reflejarse con el bajo rendimiento académico, si lo comparamos con el grupo de estudiantes motivados.

Figura 3

Resultado del cuestionario dimensional AANM

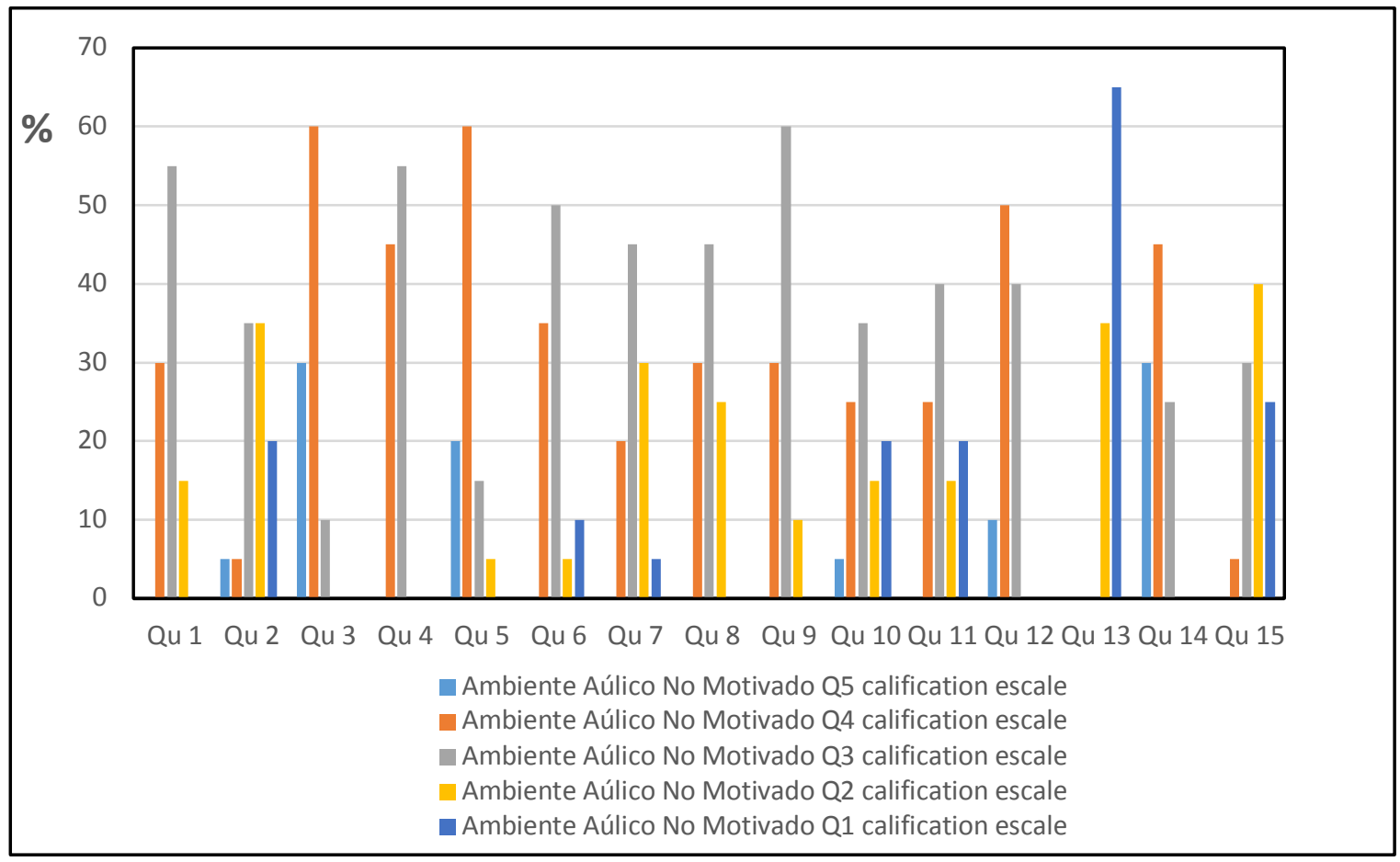

En el cuadro 2 se observan los resultados del promedio \pm desviación estándar de las respuestas al instrumento por parte de los estudiantes en AAM y AANM. En la tabla también se describe además del promedio el valor máximo o efecto techo y el valor mínimo o efecto suelo de las respuestas al instrumento de medición.

Se obtiene un efecto techo de 5 puntos (calificación más alta) para Qu1, Qu2, Qu3, Qu4, Qu6, Qu8, Qu9, Qu10, Qu11, Qu13 y Qu14 para el grupo de los estudiantes que estuvieron en el amiente áulico motivado (AAM). Mientras que para el grupo de estudiantes no motivado (GNM), se obtiene un efecto suelo de un (1) punto, para Qu 2, Qu6, Qu7, Qu10, Qu11, Qu13, Qu15. 
Fernández et al (2020), considera que existen una serie de factores intervienen en el rendimiento académico, entre esos tenemos a la motivación y el ambiente áulico de aprendizaje. Los resultados obtenidos indican que un ambiente áulico motivado, juega un rol relevante en el proceso de enseñanza aprendizaje de los estudiantes, dado que estos pueden lograr un efecto positivo, con los cuales se obtiene un rendimiento académico superior, contrario a como sucede en ambientes áulicos carentes del factor motivacional (Feng et al, 2019; Sukor et al, 2017).

\section{Cuadro 2}

Promedio de respuesta

al Instrumento IAAMA.

\begin{tabular}{|c|c|c|c|c|c|c|}
\hline \multirow{2}{*}{ Dimensión $\left(Q u^{*}\right)$} & \multicolumn{2}{|c|}{ Promedio $\pm D S$} & \multicolumn{2}{|c|}{ Efecto techo } & \multicolumn{2}{|c|}{ Efecto suelo } \\
\hline & MG & NMG & MG & NMG & MG & NMG \\
\hline 1. Qu1 ¿Qué tanto le agradó la clase? & $4,15 \pm 0,67$ & $3,15 \pm 067$ & 5 & 4 & 3 & 2 \\
\hline 2. Qu2 ¿Se sintió cómodo durante la clase? & $4,50 \pm 0,61$ & $2,4 \pm 1,05$ & 5 & 5 & 3 & 1 \\
\hline $\begin{array}{l}\text { 3. Qu3 ¿considera que el ambiente áulico es motivacional para el } \\
\text { aprendizaje de la asignatura? }\end{array}$ & $4,30 \pm 0,57$ & $4,2 \pm 0,62$ & 5 & 5 & 3 & 3 \\
\hline $\begin{array}{l}\text { 4. Qu4 ¿Sintió ganas de estar en el aula recibiendo los contenidos } \\
\text { de la asignatura? }\end{array}$ & $4,85 \pm 0,37$ & $3,45 \pm 0,51$ & 5 & 4 & 4 & 3 \\
\hline 5. Qu5 ¿Sintió sudoración durante el desarrollo de la clase? & $1,50 \pm 0,51$ & $3,95 \pm 0,76$ & 2 & 5 & 1 & 2 \\
\hline $\begin{array}{l}\text { 6. Qu6 ¿Le gustaron las herramientas y ayudas didácticas utilizadas } \\
\text { por el docente? }\end{array}$ & $4,55 \pm 0,60$ & $3,1 \pm 0,91$ & 5 & 4 & 3 & 1 \\
\hline 7. Qu7 ¿Le pareció que la clase fue aburrida? & $1,3 \pm 0,47$ & $2,8 \pm 0,83$ & 2 & 4 & 1 & 1 \\
\hline $\begin{array}{l}\text { 8. Qu8 ¿La clase fue interesante y le dieron ganas de participar } \\
\text { durante el desarrollo de esta? }\end{array}$ & $4,7 \pm 0,66$ & $3,05 \pm 0,76$ & 5 & 4 & 3 & 2 \\
\hline 9. Qu9 ¿Le gustó la dinámica de la clase? & $4,5 \pm 0,61$ & $3,2 \pm 0,62$ & 5 & 4 & 3 & 2 \\
\hline 10. Qu10 ¿Considera que el espacio del aula es amplio y adecuado? & $4,85 \pm 0,49$ & $2,8 \pm 1,20$ & 5 & 5 & 3 & 1 \\
\hline 11. Qu11 ¿La temperatura dentro del aula fue agradable? & $4,65 \pm 0,59$ & $2,7 \pm 1,08$ & 5 & 4 & 3 & 1 \\
\hline $\begin{array}{l}\text { 12. Qu12 ¿El nivel de ruido dentro del aula causó alguna } \\
\text { distracción? }\end{array}$ & $1,55 \pm 0,60$ & $3,7 \pm 0,66$ & 3 & 5 & 1 & 3 \\
\hline 13. Qu13 ¿Existió interacción grupal con sus compañeros de aula? & $4,55 \pm 0,51$ & $1,35 \pm 0,49$ & 5 & 2 & 4 & 1 \\
\hline $\begin{array}{l}\text { 14. Qu14 ¿Considera que la interacción grupal, lo motiva para el } \\
\text { aprendizaje? }\end{array}$ & $4,75 \pm 0,64$ & $4,05 \pm 0,76$ & 5 & 5 & 3 & 3 \\
\hline 15. Qu15 ¿Considera que el ambiente áulico es el a adecuado? & $4,5 \pm 0,69$ & $2,15 \pm 0,88$ & 5 & 4 & 3 & 1 \\
\hline
\end{tabular}

n = 20; MG: grupo de estudiante en ambiente aúlico motivado;

NMG: Grupo de estudiante en ambiente áulico no motivado.

Fuente: los autores

El análisis de los resultados, proporciona evidencia de los efectos positivos de la percepción que tengan los estudiantes sobre el aula y el entorno de aprendizaje, de tal manera que tengan la capacidad, de adoptar enfoques profundos para el aprendizaje. De tal manera que el ambiente áulico permita en los estudiantes un grado de motivación, y así, el proceso de enseñanza aprendizaje, sea más fluido y satisfactorio (Poondej \& Lerdpornkulrat, 2016). Las características del ambiente áulico adecuado, permiten tener escenarios reales donde se puede constatar la aplicación de los conocimientos y habilidades adquiridas.

El rol del docente, también es importante para lograr motivación en los estudiantes, y esta hace referencia a la tarea principal que se tiene, es propiciar la generación de ambientes áulico de aprendizaje que favorezcan el clima del salón de clases favoreciendo en todo momento la adquisición de competencias por parte de los alumnos, de ahí que debemos establecer el ambiente adecuado para el desarrollo de las actividades de enseñanza-aprendizaje, atendiendo a las características de cada uno de los alumnos que conforman el grupo, 
tarea nada fácil para el docente, pues los tipos de aprendizaje de cada uno de ellos es diferente, pero es una gran herramienta para el facilitador conocer a su grupo para que utilice recursos que requieren para su efectivo desarrollo.

Es indispensable que el proceso de aprendizaje se desarrolle en un ambiente, en donde los estudiantes estén motivados, para lograr esta motivación es necesario conjugar muchos aspectos, tanto humanos como físicos. El ambiente áulico incluye algunos aspectos como el nivel de ruido, la temperatura, el espacio y la interacción grupal, por mencionar algunos, que pueden jugar un papel importante el proceso de aprendizaje y por lo tanto en el rendimiento académico.

Espinoza \& Rodríguez, (2017) plantean que, la interacción grupal, permite el desarrollo de la socio-habilidad como una competencia altamente lograda, lo que implica que se construye un clima de aprendizaje, favorable para promover el aprendizaje colaborativo, ya que muestran disposición a compartir a través de la convivencia mediante valores aceptables para asegurar la armonía en grupo.

Aunque la mayoría de los estudiantes concedan muy poca importancia al ambiente áulico de aprendizaje, en esta investigación se muestra que, estos factores influyen en el rendimiento académico. Por tanto, el espacio del aula debe de cumplir una serie de condiciones que favorezcan el proceso de enseñanza y así, el rendimiento académico. La iluminación del aula es otro factor importante a tener en cuenta, es preferible que la luz, sea de tipo natural, y que penetre por el lado contrario, de la mano con la que se escribe. De esta forma, la luz se recibirá por la derecha si el estudiante es izquierdo, y por la izquierda si es diestro (evitando la formación de sombras). Cuando sea necesario el uso de luz artificial se aconseja que se haga con una luz ambiental y otra luz que dé directamente, a la silla del estudiante. La luz ha de estar distribuida de forma homogénea, evitando los contrastes de luce y sombras. Con relación a la temperatura, algunas investigaciones recomiendan realizar actividades cognitivas a temperaturas, situadas entre los 18 y los $22{ }^{\circ} \mathrm{C}$. Por debajo de esta temperatura el frío provocaría inquietud o nerviosismo y, por el contrario, el calor provocaría somnolencia, sudor o inactividad. Tanto el frío como el calor afectan a nuestra concentración, atención y motivación. Se aconseja que la distribución de la temperatura sea homogénea.

La ventilación del aula o del espacio de estudio, es importante que se renueve periódicamente, ya que se va disminuyendo paulatinamente la proporción de oxígeno y dificulta la oxigenación del cerebro. Lo que puede provocar cierto malestar como dolor de cabeza, picor de ojos, mareos, sensación de cansancio, nerviosismo.

\section{Conclusiones}

Este estudio ha proporcionado información importante acerca de la influencia del ambiente áulico y la motivación en el rendimiento académico de los estudiantes de ingeniería de alimentos que desarrollaron el curso de análisis de alimentos. El ambiente áulico, es un factor determinante para lograr la motivación de los estudiantes. Los estudiantes que cursan la asignatura de análisis de alimentos en ambientes áulicos adecuados, dinámicos, confortables, espaciosos, cómodos, de buena iluminación y temperatura, alcanzan un grado de motivación que les permite mayor rendimiento académico, que aquellos, que lo hacen en ambientes áulicos no motivacionales.

\section{Referencias bibliográficas}

Bakadorova, O., \& Raufelder, D. (2016). Do socio-motivational relationships predict achievement motivation in adolescents with high and low school self-concepts? The Journal of Educational Research, 109(3), 219231. Recuperado de: https://doi.org/10.1080/00220671.2014.942031. 
Bukhari, T. Z., Khan, J., Shahzadi, I., \& Khalid, A. (2014). Mediating role of motivation to learn in determining elearning outcomes: a conceptual study. Business and Management, 6(2), 179-189. Recuperado de: http://ijibm.site666.com/IJIBM_Vol6No2_May2014.pdf\#page=184

Duarte, J. (2003). Ambientes de aprendizaje. Una aproximación conceptual. Revista Iberoamericana De Educación, 33(1), 1-18. Recuperado de: https://doi.org/10.35362/rie3312961

Espinoza, L., \& Rodríguez, R. (2017). Learning environments generation: analys of youth perception. Revista Iberoamericana para la Investigación y el Desarrollo Educativo, 7(14), 1-23. Recuperado de: https://doi.org/10.23913/ride.v7i14.276

Eccles, J. S., \& Midgley, C. (1989). Stage-environment fit: Developmentally appropriate classrooms for young adolescents. In C. Ames, \& R. Ames (Vol. Eds.). Research on motivation in education, 3, 139-186.

Feng, Y., Bruhn, C., Elder, G., \& Boyden, D. (2019). Assessment of Knowledge and Behavior Change of a High School Positive Deviance Food Safety Curriculum. Journal of Food Science Education, 18, 45-51. Recuperado de: https://doi.org/10.1111/1541-4329.12157

Fernández, A., Quiñones., \& Álvarez, T. (2020). Estilo de vida y rendimiento académico en Farmacología de los estudiantes de Estomatología. Revista Habanera de Ciencias Médicas, 19(3), 1-12. Recuperado de: http://www.revhabanera.sld.cu/index.php/rhab/article/view/3012

Hall, M., Ramsay, A., \& Raven, J. (2004). Changing the learning environment to promote deep learning approaches in first-year accounting students. Accounting Education, 13(4), 489-505. Recuperado de: https://doi.org/10.1080/0963928042000306837

Harsel, M., Hoogerheide, V., Verkoeijen, p., \& Gog, T.v. (2019). Effects of different sequences of examples and problems on motivation and learning. Contemporary Educational Psychology, 58, 260-275. Recuperado de: https://doi.org/10.1016/j.cedpsych.2019.03.005

Hernández, A.S., Torres, F., Fang, L.C., \& Díaz, A. (2017). Estrategias de aprendizaje en estudiantes de odontología de una universidad pública en Cartagena, Colombia. Univ Odontol, 36(76), 1-9. Recuperado de: https://doi.org/10.11144/Javeriana.uo36-76.eaeo.

Kulakow, S. (2020). Academic self-concept and achievement motivation among adolescent students in different learning environments: Does competence-support matter? Learning and Motivation, 70, Article 101632. Recuperado de: https://doi.org/10.1016/j.Imot.2020.101632

Lazarides, R., \& Raufelder, D. (2017). Longitudinal effects of student-perceived classroom support on motivation-A latent change model. Frontiers in Psychology, 8, 417, 1-11. Recuperado de: https://doi.org/10.3389/fpsyg.2017.00417

Lewthwaite, R., \& Wulf, G. (2017). Optimizing motivation and attention for motor performance and learning. Current opinion and psychology, 16, 38-42. Recupersdo de: https://doi.org/10.1016/j.copsyc.2017.04.005

Orsini, C., Binnie, V., Evans, P., Ledezma, P., Fuentes, F., \& Villegas, M.J. (2015). Psychometric validation of the academic motivation scale in a dental student sample. J Dent Educ, 79(8), 971-81. Recuperado de: https://pubmed.ncbi.nlm.nih.gov/26246537/

Poondej, C., \& Lerdpornkulrat, T. (2016). Relationship between motivational goal orientations, perceptions of general education classroom learning environment, and deep approaches to learning. Kasetsart Journal of Social Sciences, 37(2), 100-103. Recuperado de: https://doi.org/10.1016/j.kjss.2015.01.001

Reindl, M., Tulis, M., \& Dresel. M. (2020). Profiles of emotional and motivational self-regulation following errors: Associations with learning. Learning and Individual Differences, 77, Article 101806. Recuperado de: https://doi.org/10.1016/j.lindif.2019.101806 
Sukor, R., Mohd Ayub, A.F., Norhasnida, Z., \& Nor Khaizura, A. R. (2017). Influence of Students' Motivation on Academic Performance among Non-Food Science Students Taking Food Science Course. International Journal of Academic Research in Progressive Education and Development, 6(4), 104-112. Recuperado de: http://dx.doi.org/10.6007/IJARPED/v6-i4/3528

Wouters, A., Croiset, G., Galindo-Garre, F., \& Kusurkar, R. A. (2016). Motivation of medical students: selection by motivation or motivation by selection. BMC Med Educ, 16(1), 37. Recuperado de:

https://doi.org/10.1186/s12909-016-0560- 1

Zürcher, Z., Rodriguez, J., Jenkins, S., Keenan, K., \& Nijland, M. (2010). Performance of juvenile baboons on neuropsychological tests assessing associative learning, motivation and attention. Journal of Neuroscience Methods, 188 (215), 219-225.

\section{Anexos}

Anexo 1

Instrumento de medición motivacional tipo Likert (IAAMA)

Responder marcando con una $\mathbf{X}$, cada una de las preguntas del cuestionario, teniendo en cuenta una escala de valores de 5 criterios, de uno punto cero (1.0) a cinco puntos cero (5.0), siendo (1.0) la calificación más baja y (5.0) la calificación más alta.

\begin{tabular}{|c|c|c|c|c|c|c|}
\hline \multicolumn{7}{|l|}{ Nombre: } \\
\hline \multicolumn{7}{|l|}{ Sexo: } \\
\hline \multicolumn{7}{|c|}{ Edad: } \\
\hline \multicolumn{7}{|c|}{ Asignatura: Análisis de alimentos. } \\
\hline \multicolumn{7}{|c|}{ Cuestionario } \\
\hline $\begin{array}{l}\text { Número } \\
\left(Q u^{*}\right)\end{array}$ & Pregunta & 5 & 4 & 3 & 2 & 1 \\
\hline Qu1 & ¿Que tanto le agradó la clase? & & & & & \\
\hline Qu2 & ¿Se sintió cómodo durante la clase? & & & & & \\
\hline Qu3 & $\begin{array}{l}\text { Considera que el ambiente áulico es motivacional para el aprendizaje } \\
\text { de la asignatura? }\end{array}$ & & & & & \\
\hline Qu4 & $\begin{array}{l}\text { Sintió ganas de estar en el aula recibiendo los contenidos de la } \\
\text { asignatura? }\end{array}$ & & & & & \\
\hline Qu5 & Sintió sudoración durante el desarrollo de la clase? & & & & & \\
\hline Qu6 & $\begin{array}{l}\text { Le gustó las herramientas y ayudas didácticas utilizadas por el } \\
\text { docente? }\end{array}$ & & & & & \\
\hline Qu7 & Le pareció que la clase fue aburrida? & & & & & \\
\hline Qu8 & $\begin{array}{l}\text { La clase fue interesante y le dieron ganas de participar durante el } \\
\text { desarrollo de esta? }\end{array}$ & & & & & \\
\hline Qu9 & Le gustó la dinámica de la clase? & & & & & \\
\hline Qu10 & Considera que el espacio del aula es amplio y adecuado? & & & & & \\
\hline Qu11 & La temperatura dentro del aula fue agradable? & & & & & \\
\hline Qu12 & El nivel de ruido dentro del aula causó alguna distracción? & & & & & \\
\hline Qu13 & Existió interacción grupal con sus compañeros de aula? & & & & & \\
\hline Qu14 & Considera que la interacción grupal, lo motiva para el aprendizaje? & & & & & \\
\hline Qu15 & ¿Considera que el ambiente áulico es el adecuado? & & & & & \\
\hline
\end{tabular}

*Qu: (Dimension). IAAMA: instrumento de medición motivacional en ambiente áulicos.

Fuente: Los autores

Esta obra está bajo una Licencia Creative Commons

Attribución-NoCommercial 4.0 International

(cc) EY-NC 